\title{
A STUDY OF VEIN RECOGNITION SYSTEM
}

\author{
Tarshi Jain $^{1}$, Rajendra Kumar $^{2}$ \\ ${ }^{1}$ M. Tech. Scholar \\ 1,2Vidya college of Engineering, Meerut \\ Corresponding Author Email: tarshijain@vidya.edu.in, rajendra.kumar@vidya.edu.in
}

This is an open access article distributed under the Creative Commons Attribution License, which permits unrestricted use, distribution, and reproduction in any medium, provided the original work is properly cited

\section{ARTICLE DETAILS}

\section{Article History:}

Received 15 November 2018 Accepted 17 December 2018 Available online 8 February 2019

\section{ABSTRACT}

The vein recognition is the most accurate and secure technology in the field of biometrics. We have seen that many criminal cases like risk of forgery or theft. All biometric traits have their own advantages and disadvantages. Like in long term, a person's fingerprint may be damaged due to environment, aging or ethnicity; face recognition has medium accuracy; iris recognition is costly affair. To overcome all the problems, the solution is vein recognition. The most important point about vein recognition system is that it works on living persons only as the infrared radiation is absorbed by hemoglobin present in blood of living persons. The skin integrity does not affect the accuracy or readability of finger vein recognition. There are lots of benefits to include vein recognition in biometric traits in which some are, users are not required to get in physical contact with the device. This technology can also be used for medical purposes like children that are below the age of 12 have very smooth vein which sometime cannot be detected by doctors to inject injection which can be detected by NIR cameras. In this paper we have presented the various techniques of palm vein recognition that are applied in today's scenario, Need and Scope of vein recognition system, its implementation challenges, its application, Merit and Demerit and finally conclusion.

\section{KEYWORDS}

Pattern Recognition, Biometrics, Image processing, Medical imaging, Security

\section{INTRODUCTION}

Vein recognition is the most accurate and reliable biometric system in the upcoming years. It is most recent and latest field. Researchers give attention to this field as this recognition system is reliable, unique \& secure. In addition, the veins are present underneath of the skin which is very special feature for personal identification. This system has many advantages over other biometric traits, some of them are: first, it is a contactless technology means the device that captures images of veins is not physically touch with the skin. Second, it only works on the alive person means if the person is dead that means his/her vein is also dead and nobody can use the vein biometric of another person for his/her counterfeit purpose. It is also a cost-effective technique. The hardware required is the NIR camera of wavelength $750 \mathrm{~nm}$ and we need software support to capture the images of hand vein and to process for recognition and identification.

\section{NEED AND SCOPE}

Starting from big organization to a particular individual realizes the advantages of biometric security for protecting the work of computers, servers, business assets etc. Nobody would want that unauthorized person to use their safe system. Due to compliance reason it is necessary to ensure the dependable process followed. Every creature is unique and carries a separate identity in the form of traits like fingerprint, iris, face geometry, voice etc. but these systems are easily spoofed by the hackers. There is a greater need of the system which is not easily cracked or spoofed. However, some cases have been reported in vein recognition spoofing [9]. With the powerful feature of vein recognition, the future of this technology is very robust. However, the limitation of passwords, PINS, biometric traits like fingerprint [11], iris, etc. weakens the security system as to break these passwords and PINS is comparatively easy. With rapid innovation and development processes getting more convenient \& secure, so that one can access their ATMs, smart card, mobile phones safely. Moreover, this system also help in taking attendance in organization, help in surveillance. It can also implement in e-commerce, in cloud-based biometrics and so on.

\section{RELATED WORK}

A scholar presented "Vein Pattern Recognition: A secured way of Authentication". In their paper, they presented a review of palm vein recognition, its working principle and development, its applications, advantages and disadvantages of the vein recognition system. According to them, vein recognition system is secure and effective technology that helps keep the information safe. As the palm vein of person is unique, this feature can be used for identification of a particular person. They mention that according to Fujitsu, comparisons of 140000 palms have been done and the result was found TFA (total false acceptance rate) found less than $0.00008 \%$. Further they explained the application of this recognition system as it is used in banking, hospitals, government offices, ATM etc. Also, they have described the pre-processing method in which some steps are ROI segmentation, Image Enhancement, Feature extraction and Pattern matching. In last, they conclude that there are many palm vein recognition techniques which are already been developed but new technique with more additional feature can improve system if this system is introduce in our country then it can solve many problems of frauds, hacking, theft, infringement, forgery, etc. This will bring a big rebellion in 
the future [1].

In a study, a scholar presented "A Study of finger vein Biometric for personal identification". Finger vein is the secure and convenience identification method as it is located inside the body. When the finger is placed under the web camera, they found that with the vein pattern shade of muscle, bones, tissue, surrounded the vein also produce. To remove all these noises produced in vein pattern they introduce adaptive threshold method, median filtering and massive noise removal technique to improve the image quality. In their paper they conclude that since pattern was taken from web camera and image are not in good quality as compared to other high-quality camera images. They found $0 \%$ of FAR and $0.275 \%$ of FRR and $100 \%$ of authentication rate. They planned to improve the imaging device and some algorithm for better result in future [2].

A previous researcher presented "A study of hand vein recognition method" in which they gave a deep concept of new threshold segmentation and thinning method of hand vein image. End points and Crossing points were studied for feature extraction. For matching method distance was used to match the vein images. From that, they conclude that the existing algorithms were effective for recognition, but they need some further improvement while the feature extracting and matching module relatively simple [3]. Moreover, authentication or we can say biometric technology is improving gradually in a long run for the betterment of mankind.

In a study, a scholar presented "A finger Vein Recognition", as a promising biometric technology in terms of security purposes. This system only works on living person. In their paper, Neighborhood elimination technique is used to extract finger vein minutiae image. The purpose of this technique was to remove the redundant information only useful information kept for further subsequent processing. Some points were taken into consideration such as vein intersection point pattern with number of intersections. Through, that technique level of security increases in the vein biometrics [4].

In a previous research, "Hand vein recognition based on multiple key points sets". In this paper, SIFT (Scale invariant feature transform) was used for matching purpose. The proposed system reduced information redundancies which, were applied on the database of 2040 images and the result comes with a high performance of $97.95 \%$ recognition rate. Fusion of multiple sets of key points was proposed to find the most reliable features [5].

In a study "Palm vein verification system based on SIFT matching". In this, they proposed that after the preprocessing and binarization, the images of vein pattern were featured by the specific patterns. The main thing in this paper is that only single image was used for the enrollment process which makes the system more effective and less time consuming. In this research, they collected the database of 24 persons. Two sessions had been made in which 30 images for each session. Total images collected were $24 \times 30 \times 2=$ 1440 images. From that one image was used for the enrollment and other remaining images for verification. As the biometric mode is very difficult to break, it can be helpful in many authentication applications. This system is based on SIFT descriptors for the enrollment \& verification steps. The efficiency of this system is very high. They obtain EER value $0.14 \%$ and ideal EER value achieved as $0 \%$ [6].

In a study "personal recognition based on dorsal hand vein pattern". In the fast-growing recognition system, hand vein biometric security system offers a reliable system for the authentication and identification. They proposed that the NIR camera used for capturing the dorsal hand vein image and processed image acquisition, preprocessing, feature extraction and classification or matching on the captured images. According to the paper, maximum curvature was used to find the centerlines of veins when the pre-processing step was over. For matching system, simple correlation method was used. In the first phase image acquisition, they were showed absorption spectrum of oxygenated and deoxygenated hemoglobin (HB) in which they found that deoxygenated hemoglobin absorbs the light with wavelength of $760 \mathrm{~nm}$. In preprocessing ROI is determined and they also showed lower and upper boundaries of hand region. The performance of the system was high. Their database consists of right- and left-hand images of male and female both. The size was $720 \times 480$ pixels. The results were computed through Matlab 7.8 version. Experimental Results shows a positive detection rate of $75 \%$ and negative detection rate is $25 \%$ they further conclude that this system can also be improved by applying additional recognition system [7].

In a study "A survey on palm vein recognition". In this paper they explained the different technique applied on palm vein recognition up till now and what improvement will have to be done for future scope. As society need a reliable personal recognition system through which it can be determine or confirm their personal identity. NIR camera was used to capture the vein of the human that resides underneath of the skin and the blood vessels absorb the infrared lights and illuminates dark. The palm vein recognition system is start from image acquisition and ended up to matching system. They further explained the contactless palm vein recognition using mutual foreground based local binary pattern with the help of OTSU algorithm and Gaussian blur is used for smoothing of images. Next, they explained feature extraction method in which they describe 2 module: online biometric authentication using hand vein pattern and biometric authentication based on infrared thermal hand vein patterns in which they find two approach to find the vein pattern i.e. branch point and box approach. If there are more than two paths starting from that point in vein pattern it is said to be branch point and in box approach, image is divided into equal size boxes and then their orientation and distance from the left corner is calculated in terms of features and then these features is compared with other two input images. So, these are the surveys in their paper, they also mentioned that there still there is need of further improvement in the field of vein recognition system [8].

As per one company name Fujitsu ltd. received an order from two Japanese banks that works on vein recognition when the palm or finger is placed in the near infrared camera the scanner take the snapshot of the image. The data collected is loaded to the server or it is designed in the form of IC cards and used as an ATM. The Bank of Tokyo-Mitsubishi Ltd., one of the biggest banks of Japan that install ATMs that works on vein recognition technology [9-11].

\section{VEIN RECOGNITION SYSTEM SETUP}

\subsection{NIR Camera}

Near infrared camera are very useful for scanning images. These cameras are used to capture the image data that lie under near-infrared spectrum. NIR cameras are imaging cameras that are designed with certain sensor coating that can detect the near infrared light. These cameras have the additional feature that it can see through certain obstacles that cannot even accomplish with the standard visible spectrum imaging cameras or are invisible to the naked eyes. NIR camera are very popular for many applications that need to utilize this wavelength range, examples are poor light condition of some applications, such as traffic monitoring. Until now, these applications were only possible with expensive CCD sensors. New CMOS technology can do the job, providing sensors with increased sensitivity in the near-infrared range over $850 \mathrm{~nm}$. Some application needs NIR camera for high wavelength as well as for normal lightning, some advantages of NIR camera are: it has very good sensitivity in the infrared range, it gives very high contrast image even in difficult illumination, it has favorable price as compared to CCD cameras, and it gives high quality results.

\subsection{Vein viewer}

It is a system to observe veins it uses infrared light to look under the skin it can then project an HD image of the vein on to the surface of the skin. This device enables the doctor and nurses to find a vein immediately. So, hospitals in the US are trailing a device that can locate inside a person's arm using harmless near infrared light called vein viewer and then projects the image of them in real time on top of the arm to help the medical worker know where to put the needle.

\subsection{Dual Camera}

Dual Camera means that instead of just one lenses, there are two lenses which help in capturing or shooting. Dual Camera is what the name suggests, two cameras together. It means two lenses, two sensors, everything two. Dual camera phones feature use two cameras including a primary camera and a second primary camera. Primary camera does the same work or with little modification as it does in typical Smartphone while the second primary camera normally has two features. The first is to add clarity of the image with the help of monochrome feature and the 
second is for zooming purpose. And sometimes in a dual lens camera setup one camera is used to take the pictures and the other one is to capture the depth of field.

\section{IMPLEMENTATION CHALLENGES}

Some implementation challenges that have been found in the study of vein recognition system those are: When an image is captured, researchers found some issues related to the position of camera and position of hand. There is a need to fix the position of camera and hand so that image of vein is captured without any noise and disturbance. Second challenge is only near infrared camera is used in these types of vein recognition research far infrared cameras are not in use for these practices.

\section{APPLICATIONS}

\subsection{Medical imaging}

It is a technique in which internal parts of the human body is clinically analysis and create visual representation of body organs and tissue. Sometimes doctor have not found the patient's vein, using this technology doctor can inject drip to the patient. Like this vein recognition system helpful in the field of medical imaging.

\subsection{Biometric Recognition for Authentication}

This technology can be helpful in the biometric recognition authentication purposes. One can unlock their personal information or access confidential data with the help of unique vein structure security system.

\subsection{Biometric Attendance}

This system can also be used for biometric attendance purpose in colleges, schools, institutions and organizations as the system is very accurate. This will also increase the security and productivity of the institution. When the enrolled person put their hand in front of near infrared camera, it captures the image and matched with the image that is pre-registered in the database.

\subsection{ATM}

Using this service of vein recognition, the customer does not need any type of smart card and pass book for their money transaction. Chances of frauds is minimized as non-registered user cannot withdrawal the money and cannot access others account.

\section{MERITS AND DEMERITS}

There are many advantages of vein recognition system in real time applications and in security systems. Some of them are firstly; this system gives a hygienic behavior as the device used to capture the images is contactless. It gives more accurate result in terms of FAR and FRR up to $0.00008 \%$. This technology is cost effective because the hardware and software required are manageable. It completely works on living person as the hemoglobin present in the veins absorbs the infrared light and vein gets darken when the image is captured by NIR camera, these all features of vein recognition system make this technology a prominent, dynamic, high performance and a secure way to keep their identity safe. While the demerit of the system is that, if a criminal or thief forcedly vandalism with anyone then only this system fall so there is a need of one more security system.

\section{CONCLUSION}

There are many different techniques that have been used in the field of vein recognition system and it has been updated regularly with new concept and with more accuracy. But there is a scope of further refinement, a new technique with more attribute, more reliability and with more certainty. If this technology put in place in India then we can solve many problems of hacking, forgery, robbery etc. It is much more beneficial if it is used under dual authentication. This technology will bring to take our country to the greater extends in terms of security and safety.

\section{REFERENCES}

[1] Kaur, N., Chopra, P. 2016. Vein Pattern Recognition: A Secured Way of Authentication, International Journal of Engineering and Computer Science, 5(10),18377-18383.

[2] Mulyono, D., Jinn, H.S. A Study of Finger Vein Biometric for Personal Identification, IEEE Xplore, ISBN: 1-4244-2427-6.

[3] Ding, Y.H., Zhuang, D.Y., Wang, K.J. A Study of Hand Vein Recognition Method, Proceedings of the IEEE International Conference on Mechatronics \& Automation Niagara Falls, Canada, 2107-2110.

[4] Brindha, S. 2017. Finger Vein Recognition, International Research Journal of Engineering and Technology (IRJET), 04(04),1298-1300.

[5] Wang, Y.D., Fan, Y., Liao, W.P., Li, K.F., Shark, L.K., Varley, M.R. Hand Vein Recognition Based on Multiple Keypoints Sets, IEEE international conference, ISBN: 978-1-4673-0397-2, 367-371.

[6] Ladoux, P.O., Rosenberger, C., Dorizzi, B. 2010. Palm Vein Verification System Based on SIFT matching, Proceedings of Third International Conference, ICB 2009, Alghero, Italy, Springer, 1290-1298.

[7] Naidile, S., Shrividya, G. 2007. Personal Recognition Based on Dorsal Hand Vein Pattern, International Journal of Innovative Research in Science, Engineering and Technology, 4(5),3189-3196.

[8] Swati, K.J., Kamble, K.P. 2016. A Survey on Palm Vein Recognition, International Journal of Advanced Research in Electrical, Electronics and Instrumentation Engineering, 5(10),7859-7861.

[9] Nguyen, D.T., Yoon, H.S., Danh, T.Y., Pham, Park, K.R. 2017. Spoof Detection for Finger-Vein Recognition System Using NIR Camera, 2261(17),1-34.

[10] Watanabe, M. 2008. Palm Vein Authentication, Advance in biometrics, Springer Link, 75-88.

[11] Kumar, R., Gupta, J.K. 2010. An efficient ANN Based approach for Latent Fingerprint Matching, International Journal of Computer Application, Foundation of Computer Science, USA, ISSN: 0975-8887, $7(10), 18-21$. 